\title{
Stress and eating behavior
}

\section{Achim Peters $^{1 *}$ and Dirk Langemann ${ }^{2}$}

\author{
Addresses: ${ }^{1}$ Brain Metabolism, Neuroenergetics, Obesity and Diabetes, Medical Clinic 1, Ratzeburger Allee 160, D-23538 Lübeck, Germany; \\ ${ }^{2}$ Computational Mathematics, Carolo-Wilhelmina University of Braunschweig, Pockelsstraße 14, D-38106 Braunschweig, Germany \\ *Corresponding author: Achim Peters (achim.peters@uk-sh.de) \\ FI000 Biology Reports 2010, 2:13 (doi:10.3410/B2-13)
}

The electronic version of this article is the complete one and can be found at: http://fl $000 . c o m /$ reports/biology/content/2/I3

\begin{abstract}
How stress, the stress response, and the adaptation of the stress response influence our eating behavior is a central question in brain research and medicine. In this report, we highlight recent advances showing the close links between eating behavior, the stress system, and neurometabolism.
\end{abstract}

\section{Introduction and context}

When humans undergo acute mild mental stress, their global cerebral metabolic rate for glucose increases by $12 \%$ [1]. As the brain has limited storage capacity, the cerebral energy demand from the body should closely match the cerebral needs. That the brain is capable of controlling its energy supply could be seen from the early observation that during inanition all organs of the body (heart, liver, kidney, and pancreas) lose $40 \%$ of their weight whereas the weight of the brain changes by only $1 \%$ or less [2].

To demand energy from the body, the brain can activate its stress system (i.e., the sympathetic nervous system [SNS] and the hypothalamus pituitary adrenal [HPA] system). Once the stress networks in the upper brain stem, including the ventromedial hypothalamus (VMH) and the paraventricular nucleus, are activated, energy particularly glucose - is allocated to the brain. With SNS activation, insulin secretion from beta cells is suppressed [3] and insulin-dependent glucose uptake via glucose transporter type 4 (GLUT4) into the body periphery becomes limited $[4,5]$. In this way, glucose is available via insulin-independent GLUT1 transport across the blood-brain barrier $[6,7]$.

The failure of the brain to match its energy demand with its need becomes evident in patients with Addison's disease. Addison patients, who are known to display disintegration of the entire stress system, have been shown not only to lack sympathoadrenal activity but also to suffer from neuroglycopenic symptoms ('neuroglycopenia' refers to a shortage of energy [glucose] in the brain) [8]. It is noteworthy that this deficit in cerebral energy could be alleviated partly by the intake of high-calorie food [8].

As excess cerebral glucose uptake has been observed during acute mental stress in humans [1], subsequent animal studies have provided evidence that functional brain activation leads to cerebral accumulation of products in intermediary metabolic pools that are subsequently consumed and oxidized during recovery [9].

After acute stress, energy homeostasis should be restored. The excess glucose extracted by the brain has to be replenished in the body stores. One possible solution is taking up energy from the near environment (i.e., eating food) [10]. The SNS/HPA response and the eating response to stress are sequentially activated, with glucocorticoids being crucial in integrating these modes of energy procurement [11]. These two stress responses are finely tuned and adapted in order to match the exogenous and endogenous energy fluxes toward the brain. Almost certainly, this tuning and adaptation process is executed by the stress and emotional networks located in the cerebral hemispheres.

\section{Major recent advances \\ The logistics of brain energy}

In 2009, a paper was published making use of supply chain principles and laws, taken from the fields of logistics 
Figure I. Supply chain of the human brain

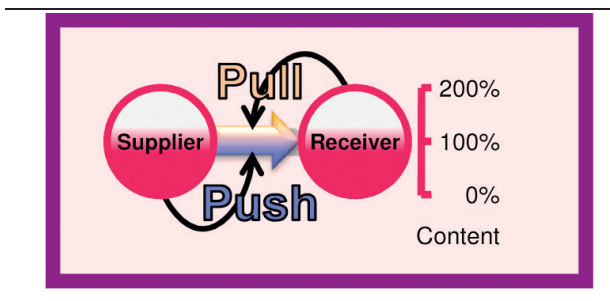

\section{Normal}

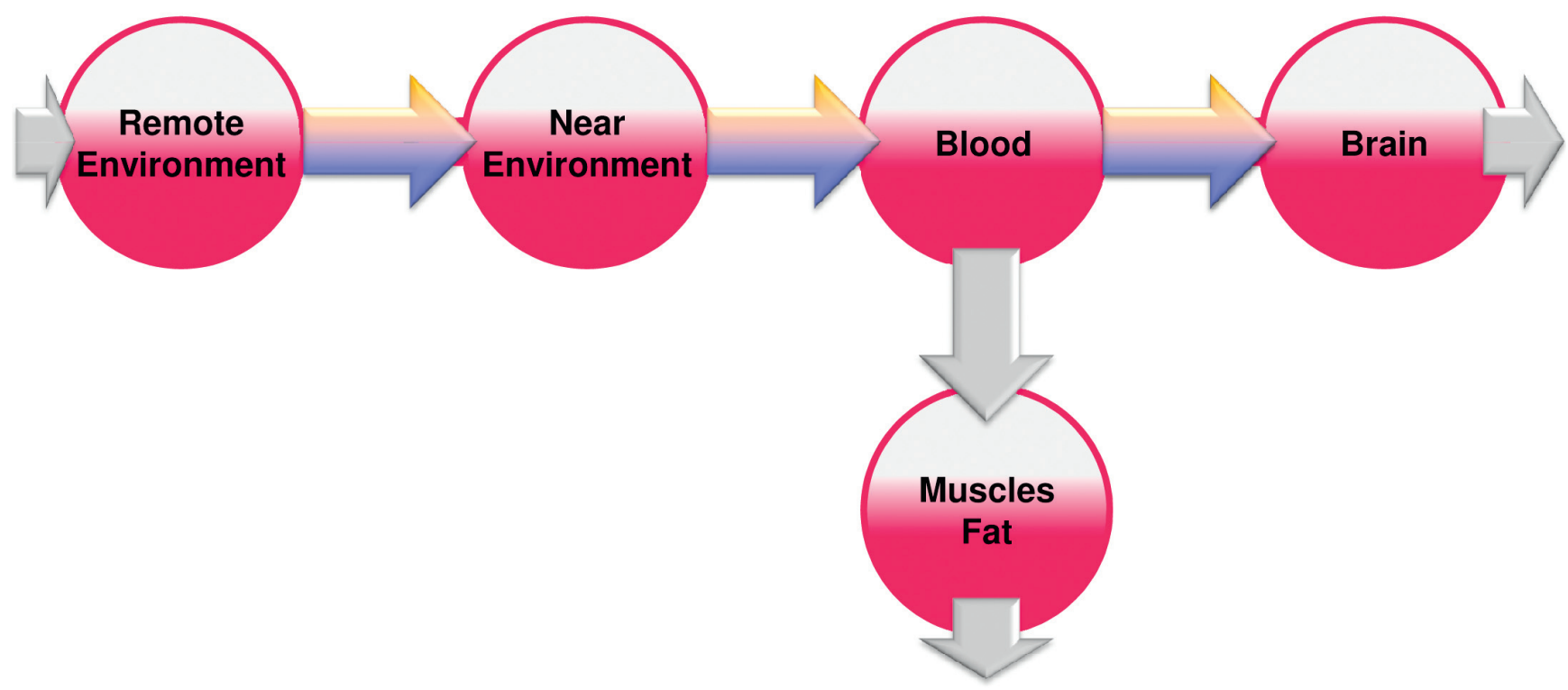

Energy from the remote environment is brought to the immediate environment, it is then taken up by the body, and from there a large part of it enters the brain. In a general supply chain, the flux of energy is determined principally by the supplier (previous step) and the receiver (proximate step). Insert: The share of the flux which is determined by the supplier is called the 'push component' (blue part of the arrows); the share which is determined by the receiver is called the 'pull component' (yellow part of the arrows).

and economics, to describe the central and peripheral energy metabolism [12]. The supply chain of the brain with the central nervous system as the final consumer describes the energy fluxes from the remote environment to the near environment, through the body, toward the brain (Figure 1). The supply chain is branched; that is, it is possible to store energy in side buffers such as muscle and fat tissue. It is a general principle in economic supply chains that the flux can be determined by both the supplier (push component) and the receiver (pull component). In other words, the fluxes are regulated by offer and demand (insert in Figure 1). The fluxes transport the energy in various forms. This publication on neuroenergetics presented the concept that a healthy organism is maintained by an 'efficient brain pull' which serves systemic homeostasis and that the underlying cause of obesity is 'inefficient brain pull' (i.e., that the brain is unable to properly demand and receive glucose from the body).

\section{Brain-pull mechanisms}

In the last two years, the mechanisms of brain pull have been better understood due to major scientific contributions. The brain pull functions to demand energy from the body. Two brain-pull mechanisms have been detected so far: first, allocative brain-pull mechanisms, which activate the SNS and the HPA axis to favor glucose allocation to the brain, and, second, astrocytic mechanisms, which enhance glucose transport via GLUT1 through the blood-brain barrier.

Two new papers showed that the cerebral need for energy is sensed and metabolically assessed by intracellular ATP. 
On the one hand, Sherwin and colleagues [13] reported that activation of the SNS in the glucose-deficient state is mediated by ATP-sensitive potassium channels that are located on GABAergic neurons adjacent to VMH neurons. On the other hand, Carruthers and colleagues [14] demonstrated that the GLUT1 pore is not a fixed pore allowing glucose to enter cells but that this transport molecule displays an intercellular ATP-binding site that opens up the GLUT1 pore when intracellular ATP is absent. The discovery of this mechanism demonstrates that astrocytes which are equipped with GLUT1 are able to actively demand or 'pull' energy according to their needs. It has further been shown that astrocytes connect among each other to form astrocytic networks that supply neurons on demand [15]. In summary, mechanisms have been recently detected that fulfill a brain-pull function and allow the brain and its neurons to systemically and locally order more energy in an ATP-dependent manner.

\section{Ingestive-pull and storage-push mechanisms}

Besides the brain-pull mechanisms, there is an additional pull mechanism that conveys the demand for energy from the near environment: eating. It is not surprising that eating behavior, which constitutes a distinct step in the cerebral supply chain, is controlled by energy sensors located in a different compartment. Denis Burdakov and colleagues [16] showed that glucose receptors are found on the surface of neurons in the lateral hypothalamus and that these drive eating behavior by sensing glucose in the cerebral extracellular space. Thus, astrocytic and neuronal mechanisms driven by intracellular ATP fulfill the function of brain pull while mechanisms driven by extracellular cerebral glucose, which is closely related to blood glucose, fulfill the function of ingestive pull.

Recently, an essential work was published on how energy is stored in the side buffers of the supply chain (i.e., muscle and fat tissue) and how feeding behavior is affected. Insulin-deficient rats were provided with different amounts of insulin. The researchers could clearly show that insulin promotes body weight gain and increases the ingestion of sucrose but not fat [17]. In this way, it becomes clear that insulin fulfills the function of a storage hormone by mediating a push component of the energy flux from blood to the energy stores: the higher the energy content in the blood (glucose), the more insulin is secreted in order to enhance glucose uptake via GLUT4 into peripheral tissues. In all, recent research has expanded our knowledge of brain pull and other push/pull components that are operative in the supply chain of the brain.

\section{Stress needs and brain pull}

By means of the invasive Kety-Schmidt technique in humans, global cerebral metabolic rate for glucose has been found to be increased during mental stress [1]. It is likely that under stress conditions, the SNS/HPA response mediates the brain-pull function and is essentially involved in augmenting the body-to-brain energy flux. Since there is experimental evidence that the brain does actually consume the extracted excess glucose [9], it is also likely that the cerebral need is increased during and after mental stress.

When the behavior of the cerebral supply chain under conditions of increased cerebral need due to brain activation is studied, various changes can be observed (Figure 2): the brain pull is increased, the energy flux from the body toward the brain augmented, the energetic equilibrium in the blood and muscle/fat compartment burdened, and the peripheral energy stores reduced. These observations within the supply chain are similar to those that occurred in approximately $40 \%$ of students who participated in two British studies and reported decreased food intake during stress, or weight loss $[18,19]$. Thus, it is conceivable that weight loss in an individual is driven by a persistent SNS/HPA stress response or, in other words, by a persistent overactive brain pull. There were, however, other participants (approximately 50\%) in these British studies who, in contrast, reported increased food intake during stress, or weight gain $[18,19]$. What was different in these individuals? Did they respond differently with their SNS and HPA axis to a given stressor? Is there experimental support of this latter view?

\section{Adaptation of the stress response: from brain pull to comfort eating}

People with chronic stress often develop depressive symptoms [20], and hyperactivity of the hypothalamicpituitary-adrenal axis is one of the most consistent biological findings in major depression psychiatry [21]. When the stressor persists, some people adapt with their stress responses (i.e., their SNS/HPA responsiveness decreases) whereas others do not [22]. Does the brain in some cases use a metabolic coping strategy combining 'comfort eating' (increased intake of calorie-rich foods) with an unburdening of the stress system (brain pull) and in this way allow stabilization of mood and cerebral energy homeostasis?

When the behavior of the cerebral supply chain under conditions of both increased cerebral need and decreased brain-pull efficiency is studied, a development and 


\section{Stress}

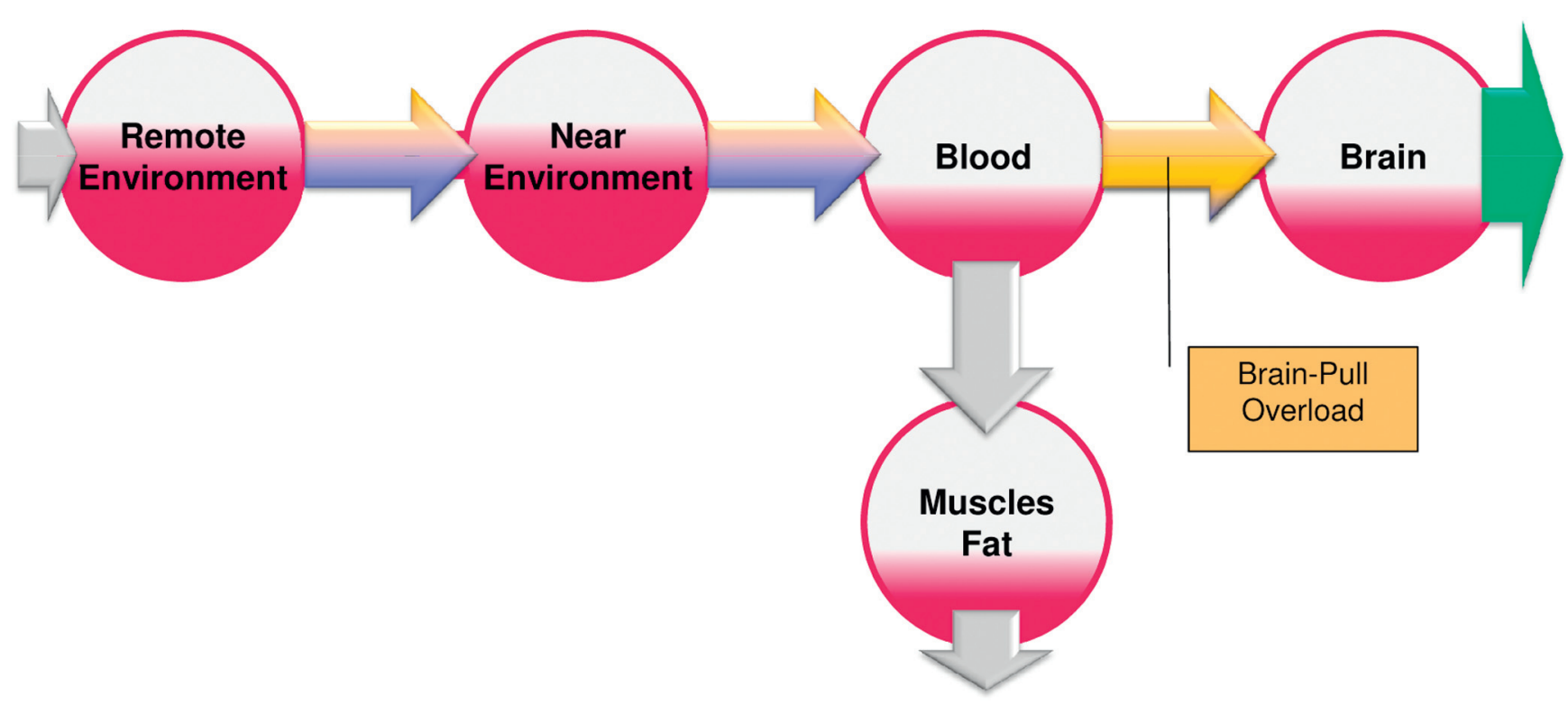

Changes are based on a simulation study using the 'supply chain' model as published in [I2]; the results are depicted in a semiquantitative manner. If the cerebral energy consumption increases (green arrow), the cerebral energy content decreases and prompts activation of the brain pull. As a consequence, energy content in the body compartment declines, particularly in muscle and fat.

progression of diverse peripheral metabolic abnormalities can be observed (Figure 3): if the needs of the brain are increased and the brain-pull activity is reduced and food intake is increased, then the brain energy content is found to be preserved. On the other hand, energy is found to accumulate in blood and peripheral tissues as a build-up in the cerebral supply chain. Under these conditions, the brain is supplied by the increased push component from the blood, and the hyperactive brainpull can remain unburdened. It could be proven analytically that a general property is inherent in the presented cerebral supply chain: the fat compartment increases with decreasing brain-pull efficiency [12]. Hence, the development of obesity is predicted to occur with an inadequate sympathoadrenal stress response.

These observations of occurrences in the supply chain as described here are similar to those abnormalities that have been found in subjects who participated in a large epidemiological study in Norway. The intriguing paper on this large cohort by Arnljot Flaa and colleagues [23] demonstrated that low sympathoadrenal activity predicts body weight gain during an 18-year follow-up study. These researchers performed a mental stress test in 99 healthy men of normal weight. In the 18-year follow-up investigation, they found that a body weight gain could be predicted by a low sympathoadrenal response to the stress test at baseline. This study provides decisive support for the view that inadequate sympathoadrenal responsiveness to a mental stressor is a crucial causal factor for the development and progression of obesity.

What are the causal factors that reduce the responsiveness of the SNS/HPA system in people who develop obesity? Chronic stress in adult life may play a major role in adaptation of the stress response. The topical paper by Block et al. [24] provides new insights into the association between stress and long-term weight gain in a large representative cohort. These researches used a longitudinal cohort of 1355 subjects and showed that several domains of psychosocial stress were associated with weight gain over a period of 9 years in both women and men with higher body mass index. Among the people with high baseline body mass index, weight gain was associated with increasing levels of psychosocial stress related to 'job-related demands' and 'difficulty paying bills'. In men, additional factors such as 'lack of skill discretion' (variety of work and opportunity for use 
Figure 3. The effect of the adaptation of the stress response on energy metabolism

\section{Adaptation of stress response}

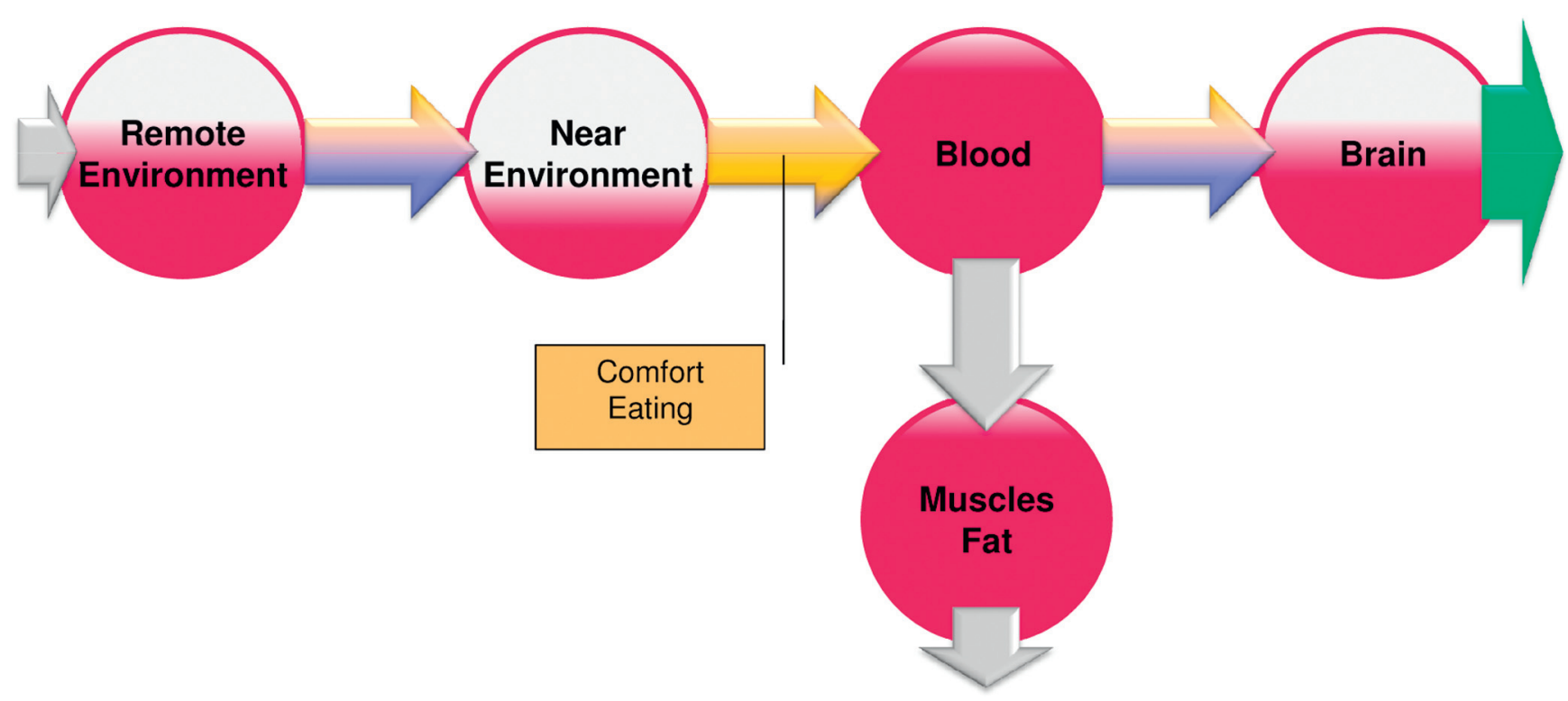

Adaptation to chronic stress may occur at two steps of the supply chain. While the brain decreases the brain pull on the one hand, it increases the ingestive pull on the other hand. In this way, cerebral energy homeostasis is maintained. However, the change of energy fluxes leads to a build-up in the supply chain, resulting in obesity and type 2 diabetes.

of skills) and 'lack of decision authority' were important; in women, 'perceived constraints in life' and 'strain in relations with family' were important. Thus, adaptation to chronic stressors could play a role in reducing brainpull efficiency and in this way promote the development of obesity.

In another study in British adults, the presence of chronic and repeated episodes of the common mental disorders of depression and anxiety was a risk factor for subsequently being overweight or obese [25]. Moreover, weight gain and the risk of obesity were increased in a dose-response fashion with the number of episodes of these common mental disorders.

Acute episodic stress in early life can also lead to adaptations in the stress response in later life. Two animal experiments have shown that early-life stress or juvenile trauma results in long-lasting changes in the activity of the autonomic nervous system and body weight $[26,27]$. In an important human study, Sonja Entringer and her colleagues [28] reported that prenatal psychosocial stress exposure is associated with hyperinsulinemia in later life. The researchers investigated healthy young adults whose mothers experienced major stressful life events during their pregnancy. As adults, these individuals displayed hyperinsulinemia, a strong predictor of weight gain $[29,30]$ and a typical marker of brain-pull inefficiency.

\section{Future directions}

The recent research papers highlighted in the present report support the view that an adaptation of the SNS/ HPA stress response to a chronic-persistent or an acutetraumatic stressor involves a strategy of the brain that on the one hand relieves the overloaded brain pull and improves mood but on the other hand makes it necessary to increase eating behavior. Thus, evidence accumulates that the stressed mind can choose a metabolic coping strategy by switching its supply mode from brain pull to 'comfort eating'.

\section{Abbreviations}

GLUT, glucose transporter; HPA, hypothalamus pituitary adrenal; SNS, sympathetic nervous system; VMH, ventromedial hypothalamus.

\section{Competing interests}

The authors declare that they have no competing interests. 


\section{Acknowledgments}

This work was supported by a grant from the German Research Foundation (Clinical Research Group KFO-126).

\section{References}

I. Madsen PL, Hasselbalch SG, Hagemann LP, Olsen KS, Bülow J, Holm S, Wildschiødtz G, Paulson OB, Lassen NA: Persistent resetting of the cerebral oxygen/glucose uptake ratio by brain activation: evidence obtained with the Kety-Schmidt technique. J Cereb Blood Flow Metab 1995, I 5:485-9I.

2. Krieger $M$ : On the atrophy of human organs during atrition. Z Angew Anat Konstitutionsl I921, 7:87-I34.

3. Fruehwald-Schultes B, Kern W, Born J, Fehm HL, Peters A: Comparison of the inhibitory effect of insulin and hypoglycemia on insulin secretion in humans. Metabolism 2000, 49:950-3.

4. Capaldo B, Napoli R, Guida R, Di Bonito P, Antoniello S, Auletta M, Pardo $F$, Rendina V, Saccà L: Forearm muscle insulin resistance during hypoglycemia: role of adrenergic mechanisms and hypoglycemia per se. Am J Physiol 1995, 268:E248-54.

5. Cohen N, Rossetti L, Shlimovich P, Halberstam M, Hu M, Shamoon H: Counterregulation of hypoglycemia. Skeletal muscle glycogen metabolism during three hours of physiological hyperinsulinemia in humans. Diabetes 1995, 44:423-30.

6. Seaquist ER, Damberg GS, Tkac I, Gruetter R: The effect of insulin on in vivo cerebral glucose concentrations and rates of glucose transport/metabolism in humans. Diabetes 200I, 50:2203-9.

7. Hasselbalch SG, Knudsen GM, Videbaek C, Pinborg LH, Schmidt JF, Holm S, Paulson OB: No effect of insulin on glucose blood-brain barrier transport and cerebral metabolism in humans. Diabetes 1999, 48:1915-21.

8. Klement J, Hubold C, Cords H, Oltmanns KM, Hallschmid M, Born J, Lehnert H, Peters A: High-calorie comfort food attenuates neuroglycopenic symptoms in patients with Addison's disease. J Clin Endocr Metab 2009, [Epub ahead of print].

9. Madsen PL, Cruz NF, Sokoloff L, Dienel GA: Cerebral oxygen/ glucose ratio is low during sensory stimulation and rises above normal during recovery: excess glucose consumption during stimulation is not accounted for by lactate efflux from or accumulation in brain tissue. J Cereb Blood Flow Metab 1999, 19:393-400.

10. Rutters F, Nieuwenhuizen AG, Lemmens SG, Born JM, WesterterpPlantenga MS: Acute stress-related changes in eating in the absence of hunger. Obesity (Silver Spring) 2009, I 7:72-7.

II. George SA, Khan S, Briggs H, Abelson JL: CRH-stimulated cortisol release and food intake in healthy, non-obese adults. Psychoneuroendocrinology 2009, [Epub ahead of print].

12. Peters A, Langemann D: Build-ups in the supply chain of the brain: on the neuroenergetic cause of obesity and type 2 diabetes mellitus. Front Neuroenergetics 2009, I:2.

13. Chan O, Lawson M, Zhu W, Beverly JL, Sherwin RS: ATP-sensitive $K(+)$ channels regulate the release of GABA in the ventromedial hypothalamus during hypoglycemia. Diabetes 2007, 56: I 120-6.

14. Blodgett DM, De Zutter JK, Levine KB, Karim P, Carruthers A: Structural basis of GLUTI inhibition by cytoplasmic ATP. J Gen Physiol 2007, I30:I57-68.

15. Rouach N, Koulakoff A, Abudara V, Willecke K, Giaume C: Astroglial metabolic networks sustain hippocampal synaptic transmission. Science 2008, 322:|55|-5.

FI000 Factor 9.6 Exceptional

Evaluated by Arturo Alvarez-Buylla 02 Jan 2009, Achim Peters 07 Jan 2009
16. Gonzalez JA, Jensen LT, Fugger L, Burdakov D: Metabolismindependent sugar sensing in central orexin neurons. Diabetes 2008, 57:2569-76.

FI000 Factor 6.0 Must Read Evaluated by Achim Peters 06 Jan 2009

17. Warne JP, Akana SF, Ginsberg AB, Horneman HF, Pecoraro NC, Dallman MF: Disengaging insulin from corticosterone: roles of each on energy intake and disposition. Am J Physiol Regul Integr Comp Physiol 2009, 296:RI366-75.

18. Serlachius A, Hamer M, Wardle J: Stress and weight change in university students in the United Kingdom. Physiol Behav 2007, 92:548-53.

19. Oliver G, Wardle J: Perceived effects of stress on food choice. Physiol Behav 1999, 66:5।I-5.

20. McGonagle KA, Kessler RC: Chronic stress, acute stress, and depressive symptoms. Am J Community Psychol 1990, 18:68I-706.

21. Pariante CM, Lightman SL: The HPA axis in major depression: classical theories and new developments. Trends Neurosci 2008, 31:464-8.

22. McEwen BS: Protective and damaging effects of stress mediators. $N$ Engl J Med I998, 338:I7I-9.

23. Flaa A, Sandvik L, Kjeldsen SE, Eide IK, Rostrup M: Does sympathoadrenal activity predict changes in body fat? An | 8-y follow-up study. Am J Clin Nutr 2008, 87:| 596-60|.

FI000 Factor 6.0 Must Read

Evaluated by Achim Peters 08 Sep 2009

24. Block JP, He Y, Zaslavsky AM, Ding L, Ayanian JZ: Psychosocial stress and change in weight among US adults. Am J Epidemiol 2009, 170: $181-92$.

25. Kivimäki M, Lawlor DA, Singh-Manoux A, Batty GD, Ferrie JE, Shipley MJ, Nabi H, Sabia S, Marmot MG, Jokela M: Common mental disorder and obesity: insight from four repeat measures over 19 years: prospective Whitehall II cohort study. BMJ 2009, 339:b3765.

26. Cohen H, Kaplan Z, Matar MA, Loewenthal U, Zohar J, RichterLevin G: Long-lasting behavioral effects of juvenile trauma in an animal model of PTSD associated with a failure of the autonomic nervous system to recover. Eur Neuropsychopharmacol 2007, I 7:464-77.

FI000 Factor 6.0 Must Read

Evaluated by Achim Peters 22 Jan 2008

27. Kaufman D, Banerji MA, Shorman I, Smith EL, Coplan JD, Rosenblum LA, Kral JG: Early-life stress and the development of obesity and insulin resistance in juvenile bonnet macaques. Diabetes 2007, 56:1382-6.

FI000 Factor 6.0 Must Read

Evaluated by Achim Peters 17 May 2007

28. Entringer S, Wüst S, Kumsta R, Layes IM, Nelson EL, Hellhammer DH, Wadhwa PD: Prenatal psychosocial stress exposure is associated with insulin resistance in young adults. Am J Obstet Gynecol 2008, 199:498-7.

FI000 Factor 6.0 Must Read

Evaluated by Achim Peters 03 Nov 2008

29. Sigal RJ, El-Hashimy M, Martin BC, Soeldner JS, Krolewski AS, Warram JH: Acute postchallenge hyperinsulinemia predicts weight gain: a prospective study. Diabetes 1997, 46:1025-9.

30. Odeleye OE, de Courten M, Pettitt DJ, Ravussin E: Fasting hyperinsulinemia is a predictor of increased body weight gain and obesity in Pima Indian children. Diabetes 1997, 46: $|34|-5$. 